\title{
Neuroimaging in Amyotrophic Lateral Sclerosis
}

\author{
Sumei Wang, Elias R. Melhem, Harish Poptani, and John H. Woo \\ Division of Neuroradiology, Department of Radiology, Hospital of the University of Pennsylvania, 3400 Spruce Street, \\ Philadelphia, Pennsylvania 19104
}

\begin{abstract}
Summary: Amyotrophic lateral sclerosis (ALS) is a motor neuron disease characterized by progressive degeneration of upper motor neurons (UMN) and lower motor neurons (LMN). While LMN dysfunction can be confirmed by electromyography (EMG) and muscle biopsy, UMN involvement is more difficult to detect, particularly in the early phase. Objective and sensitive measures of UMN dysfunction are needed for early diagnosis and monitoring of disease progression and therapeutic efficacy. Advanced magnetic resonance imaging (MRI) techniques, such
\end{abstract}

as diffusion, perfusion, magnetization transfer imaging, functional MRI, and MR spectroscopy, provide insight into the pathophysiological processes of ALS and may have a role in the identification and monitoring of UMN pathology. This article provides an overview of these neuroimaging techniques and their potential roles in ALS. Key Words: Amyotrophic lateral sclerosis, diffusion tensor, perfusion imaging, proton spectroscopy, magnetization transfer imaging.

\section{INTRODUCTION}

Amyotrophic lateral sclerosis (ALS), also known as Lou Gehrig's disease, is a fatal neurodegenerative disorder affecting upper motor neurons (UMN) in the motor cortex as well as lower motor neurons (LMN) in the brain stem and spinal cord. Patients with ALS suffer from a progressive paralysis of the skeletal muscles, resulting in loss of voluntary movement, ability to speak and eat, and eventual loss of respiratory function [1]. The motor function phenotypes of ALS are highly heterogeneous and determined by three primary independent attributes: body region of onset; relative mix of UMN and LMN involvement; rate of progression [2]. Phenotypes that predominantly affect specific body regions are associated with extremes of disease progression; for example, patients with bulbar onset are typically associated with a more rapid decline $[2,3]$. The cause of ALS is generally unknown, although approximately $2 \%$ of cases are due to mutations in the superoxide dismutase gene [4]. The disease is incurable and until now only one drug, riluzole, has been shown to be somewhat effective in prolonging the life span of patients with ALS [4].

Electronic supplementary material The online version of this article (doi:10.1007/s13311-010-0011-3) contains supplementary material, which is available to authorized users.

Address correspondence and reprint requests to: Sumei Wang, Division of Neuroradiology, Department of Radiology, Hospital of the University of Pennsylvania, 3400 Spruce Street, Philadelphia, PA 19104. E-mail: Sumei.Wang@uphs.upenn.edu.
The classical neuropathological features of ALS include loss and degeneration of the large motor neurons in the gray matter of the spinal cord, brainstem, and cortex, as well as degeneration of the corticospinal tract (CST) that contains axons of the cortical UMN. Other extramotor systems are also involved to various degrees. About $2-3 \%$ of ALS cases are accompanied by frontotemporal dementia [5, 6], while in approximately $50 \%$ of cases cognitive impairment can be observed [7].

The diagnosis of ALS is currently based on clinical features, electromyography, and the exclusion of other diseases with similar symptoms. LMN dysfunction can be confirmed by electromyography and muscle biopsy, whereas UMN involvement is more difficult to detect, particularly in the early phase $[1,4]$. Objective and sensitive measures of UMN dysfunction are thus needed since delayed diagnosis may lead to the loss of motor function, which might not be corrected by therapeutic interventions [7]. Furthermore, longitudinal studies will be required to monitor the disease progression and treatment efficacy.

In the search for the UMN markers, several neuroimaging modalities, especially diffusion tensor imaging (DTI) [8-16], proton magnetic resonance spectroscopy ( $\left.{ }^{1} \mathrm{H}-\mathrm{MRS}\right)$ [14, 15, 17-20], perfusion imaging [21], magnetization transfer imaging [22] (MTI) and functional magnetic resonance imaging (fMRI) [23-25], have been used with varying success. This article presents an overview of these neuroimaging methods and their potential roles in ALS. 


\section{CONVENTIONAL MRI}

In patients with ALS, conventional MRI is used principally to exclude other pathologies, such as cerebral lesions, skull base lesions, cervical spondylotic myelopathy, other myelopathy, conus lesions, and thoracolumbar/sacral radiculopathy $[7,26,27]$. Once diseases mimicking ALS are ruled out, subtle neuroimaging signs that are supportive of ALS are looked into.

Several studies have reported abnormal findings on T1-weighted, T2-weighted, proton-density, and fluidattenuated inversion recovery images in patients with ALS $[28,29]$. Consistent features that are indicative but not specific for the diagnosis of ALS include hyperintensity in the CST, hypointensity in the motor cortex, and brain atrophy.

\section{Hyperintensity along the corticospinal tracts}

Hyperintensity along the CST on T2-weighted or fluidattenuated inversion recovery images is most readily identified in the posterior limb of the internal capsule (PLIC) and may also be seen in the centrum semiovale and ventral brain stem [26, 27]. However, some of these findings have also been reported in normal controls [26]. The frequency of hyperintense lesions in the CST of patients with ALS varies significantly in different studies and does not correlate with clinical scores $[26,28]$.

\section{Hypointensity in the motor cortex}

Hypointensity in the motor cortex on T2-weighted images has been observed in a fraction of patients with ALS. The observed hypointensity may be due to a T2 shortening effect from excessive iron deposition [30], fibrillary gliosis, and/or macrophage infiltration [28, 31]. Theoretically, the presence of paramagnetic or ferromagnetic materials at a high enough concentration will cause susceptibility effects. Similarly, the presence of a tissue with low water content will also lead to hypointensity. However, such a change is neither sensitive nor specific to ALS pathology.

\section{Brain atrophy}

The voxel-based morphometry (VBM) approach is an automated statistical technique that has been used to detect regional differences in brain tissue density and tissue amount $[32,33]$. It has been validated as a powerful tool for analyzing changes in gray or white matter (WM) volumes in the brain [32, 34]. VBM studies in ALS patients suggest that the regional gray matter loss is not only confined to the motor cortex, but extends to the frontal, temporal, parietal, and limbic regions [3437]. The frontal regions have been reported to have the most severe atrophy in patients with ALS and frontotemporal dementia [37]. Several studies have also reported regional WM loss in ALS, including that in the corpus callosum, cerebellum, frontotemporal, and occipital regions in ALS [5, 34]. However, global brain atrophy in ALS is relatively mild [35].

\section{DIFFUSION TENSOR IMAGING}

\section{Basic physics}

Diffusion imaging probes the self-diffusional movement of water molecules, which can be detected by the attenuation of MRI signal using a diffusion weighted sequence [38]. When unimpeded, water molecules move in a random manner (isotropic diffusion). However, the presence of obstacles, such as axonal membranes and myelin sheaths in WM fiber tracts, hinders this molecular motion in a particular direction, resulting in anisotropic diffusion. Diffusivity of water is generally higher in directions along fiber tracts than in the perpendicular direction [38]. This process of diffusion can be mathematically described by a diffusion tensor ellipsoid, which is characterized by its three eigenvectors and the corresponding eigenvalues $\left(\lambda_{1}, \lambda_{2}\right.$, and $\left.\lambda_{3}\right)$. The eigenvector associated with the largest eigenvalue denotes the predominant orientation of fibers in a given imaging voxel.

The most commonly used indices for estimating diffusion are mean diffusivity (MD) and fractional anisotropy (FA) [39], which can be calculated according to Eqs. (1) and (2), respectively

$$
\begin{gathered}
\mathrm{MD}=\left(\lambda_{1}+\lambda_{2}+\lambda_{3}\right) / 3 \\
F A=\sqrt{\frac{3}{2}} \sqrt{\frac{\left(\lambda_{1}-\bar{\lambda}\right)^{2}+\left(\lambda_{2}-\bar{\lambda}\right)^{2}+\left(\lambda_{3}-\bar{\lambda}\right)^{2}}{\lambda_{1}^{2}+\lambda_{2}{ }^{2}+\lambda_{3}{ }^{2}}}
\end{gathered}
$$

where $\bar{\lambda}$ denotes the mean of the three eigenvalues.

MD is a measure of the directionally averaged magnitude of diffusion and is related to the integrity of the local brain tissue. FA represents the degree of diffusion anisotropy and reflects the degree of alignment of cellular structure [39].

DTI techniques also allow interregional fiber tracking, known as diffusion tensor tractography, which allows tracking of the major WM tracts [16, 40, 41]. This process can be carried out with a wide variety of algorithms. The commonly used streamline tractography is generally performed using a line propagation technique, which uses continuous number fields [41, 42] and multiple regions of interest (ROIs) [16, 41]. Tracking is typically launched from a "seed" voxel from which a line is propagated in both retrograde and antegrade directions according to the major eigenvector at each voxel. Tracking propagates in the direction of the eigenvector that is associated with the largest eigenvalue. The propagation terminates when it reaches a voxel with FA 
lower than a specified FA threshold or when the angle between two principle eigenvectors is greater than a specified angle threshold.

Probabilistic tractography is a relatively novel approach in which probabilistic maps of fiber connectivity between brain regions are generated. The value of each voxel in a map indicates the likelihood that the voxel is included in the diffusion path between two ROIs [40, 43]. Diffusion tensor tractography has been shown to be helpful in guiding the placement of ROIs on the CST $[15,44]$.

\section{Clinical application}

DTI can provide important measures of UMN dysfunction. Changes in tissue structure can lead to alterations in the diffusion characteristics, which can in turn be reflected by the changes in FA and MD values. Both ROI-based approaches and tractography studies have demonstrated significant changes in diffusion parameters along the CST in the brains of ALS patients [8-10, 12,
$13,15,45]$. The most common finding is a reduction of FA in the CST, which is thought to be due to the neuronal degeneration of UMN. Increased MD along the CST has been reported by some investigators [9, 12, 45, 46]; however, no such changes were observed in another study [10]. In one of these studies, Sage et al. [45] spatially interpolated FA and MD over the course of CST. FA decrease could be demonstrated at nearly all levels of the CST, with the most significant reduction in the PLIC. MD showed a more constant course over different levels of the CST and tended to be elevated at the cranial level of the CST (FIG. 1). Significant correlations of diffusion parameters with measures of disease severity and duration have been established in a number of studies [9, 10, 15], but these correlations could not be confirmed in other studies [8, 12].

Voxel-based analysis of DTI data has been used to evaluate the WM integrity in ALS [11, 45, 46]. Recently, a hybrid approach, known as tract-based spatial statistics
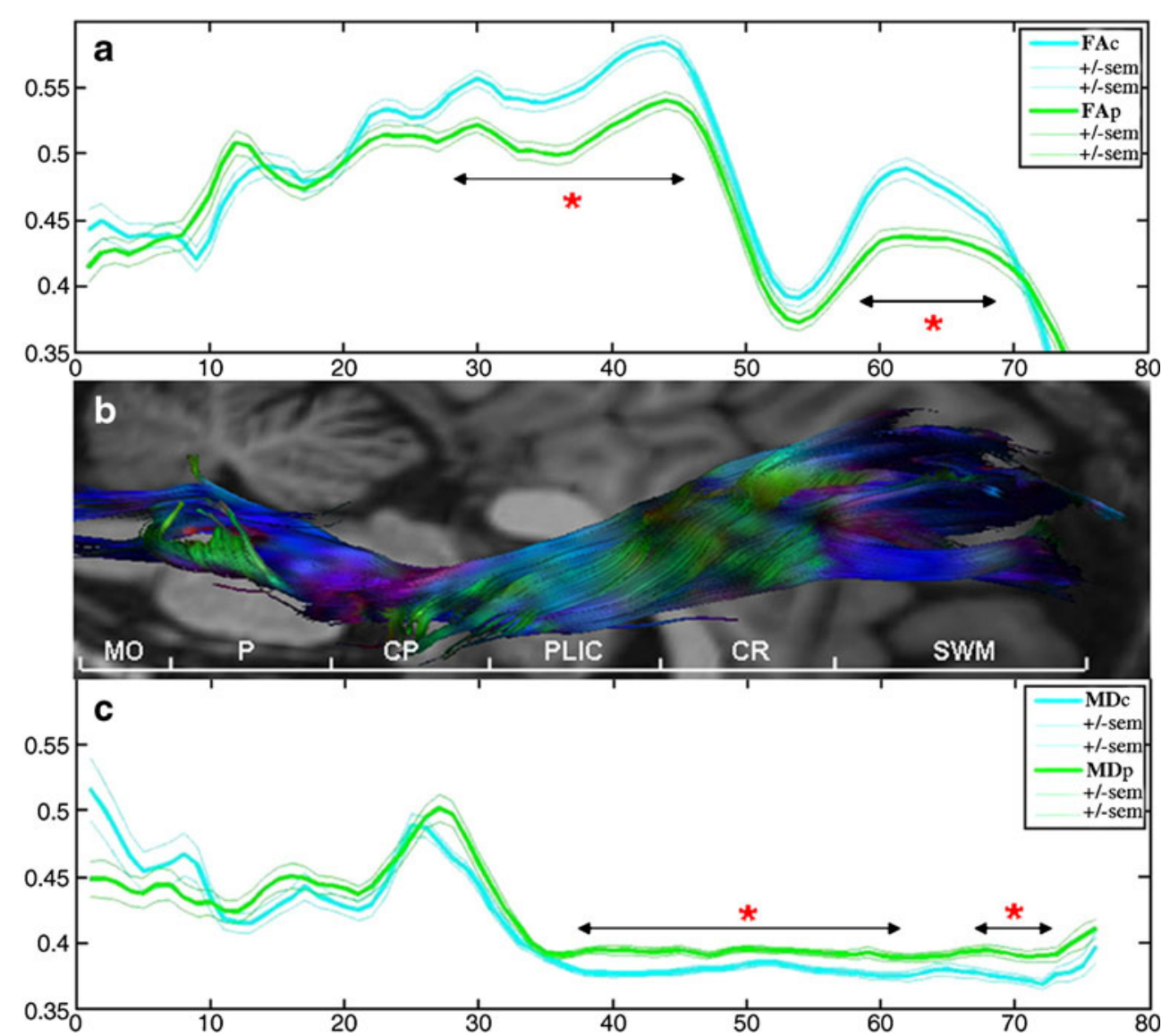

FIG. 1. Graphs of the interpolated fractional anisotropy (FA) (a) and mean diffusivity (MD) (c) values of the mean corticospinal tract (CST) in patients (green) and controls (blue). Values are shown as the mean \pm standard error (SEM). Interpolated $z$-coordinates (or slices) are shown on the $x$-axes. The $y$-axes represent values of FA and $\mathrm{MD}\left(10^{-3} \mathrm{~mm}^{2} / \mathrm{s}\right)$. Slices in which FA is significantly decreased or in which MD is significantly increased in patients compared to healthy controls are indicated by double arrows and a red asterisk. (b) A reconstructed mean CST with colors according to color encoding of DTI-based color maps overlaid on an anatomical image (flipped to match the $x$-axes of the graphs) is shown for anatomical reference, together with a vertical bar in white indicating the anatomical structures. $\mathrm{MO}=$ medulla oblongata; $\mathrm{P}=$ pons; $\mathrm{CP}=$ cerebral peduncle; PLIC = posterior limb of the internal capsule; CR = corona radiata; SWM = subcortical WM. Reprinted with permission from Sage et al. [45]. (High resolution version of this image is available in the electronic supplementary material.) 
(TBSS) [47], which attempts to combine the best of voxel-based and tract-based analyses is being increasingly used in ALS [48, 49]. A significant advantage of the TBSS approach is that it allows statistical changes to be projected on the skeleton of WM structures. Several studies have reported a significant reduction of FA not only in the CST but also in extramotor regions, including the corpus callosum, frontal and parietal WM as well as the hippocampal formation and insula [36, 45, 48, 49]. Using a different methodology, Agosta et al. [50] recently reported increased axial diffusivity of the uncinate fasciculus in ALS patients and suggested that uncinate fasciculus involvement may be related to the behavioral symptoms of patients with ALS. The results of these studies support the view that ALS is a multisystem degenerative disease in which abnormalities of extramotor areas play an important role in its pathophysiology.

\section{PROTON MR SPECTROSCOPY}

\section{Basic physics}

MR spectroscopy provides biochemical information on the tissue from a selected ROI. The MR spectrum is a spectral graph in which the $\mathrm{x}$-axis denotes the unique chemical composition of a metabolite in parts per million (ppm) and the $y$-axis reflects the concentration of that metabolite. Although the MR spectrum can be generated from any element in the periodic table that has an odd number of protons, biological MR studies are generally confined to those involving the ${ }^{1} \mathrm{H},{ }^{31} \mathrm{P},{ }^{13} \mathrm{C},{ }^{23} \mathrm{Na}$, and ${ }^{19} \mathrm{~F}$ protons. Of these, the most commonly studied is proton $\left({ }^{1} \mathrm{H}\right)$ nucleus due to its abundance in biological tissue (water and fat) and high sensitivity. Only freely mobile small molecules are detected in the in vivo MR spectrum; the signals from membrane-bound larger molecules, such as proteins, are not observed. As the tissue water concentration is about 70 molar, in comparison to the millimolar concentration of metabolites, it is critical to suppress the water signal to detect the small amount of important metabolites. This suppression of the water signal is typically achieved through the use of chemical shift selective water suppression radiofrequency pulses. Similarly, in the brain, the extra-cranial lipid (fat) signals are suppressed using outer-volume suppression techniques. In order to adequately resolve the individual metabolite resonances, it is necessary to optimize the magnetic field homogeneity within the ROI. This is achieved using a procedure called "shimming" in which the magnetic field is iteratively adjusted along the orthogonal axes of the magnet [51].

The MR spectrum in vivo can either be acquired from a single voxel using the single-voxel spectroscopy (SVS) technique or using a chemical shift imaging (CSI) method, in which multiple voxels can be studied simultaneously [27]. The SVS method requires less time than the CSI technique and provides reliable measures of metabolites from the specified ROI; however, it is limited as only a small region of the brain is interrogated at one time. The CSI method takes longer than the SVS method, as it provides spectra from an array of voxels, a single section, or multiple sections. Recently, echo planar CSI has been successfully implemented to further increase the region covered by spectroscopy studies as this technique provides metabolite distribution maps from almost the whole brain with sufficient spectral quality $[52,53]$. This technique also allows spectra to be acquired from regions that generally suffer from poor spectral quality on the SVS and CSI methods, such as the brain stem.

The major brain metabolites detected by ${ }^{1} \mathrm{H}$ MRS are $N$ acetyl aspartate (NAA), choline (Cho), creatine (Cr), myoinositol (mI), lipid, lactate, and glutamine+glutamate. It is generally accepted that NAA is a marker for neuronal integrity; Cho, a marker of membrane turnover and cell proliferation; $\mathrm{Cr}$, a marker of tissue energy state; $\mathrm{mI}$, an astrocytic marker; lipid signals, indicators of tissue destruction and necrosis; lactate, an indicator of hypoxia; glutamine+glutamate, excitatory markers [51].

\section{Clinical application}

Most of the ${ }^{1} \mathrm{H}$-MRS studies in ALS have demonstrated that either NAA concentrations [54, 55] or ratios of NAA/Cr, $[15,18,19,56] \mathrm{NAA} / \mathrm{Cho}[15,17$, 55], and $\mathrm{NAA} /(\mathrm{Cr}+\mathrm{Cho})$ [57] are reduced in the motor cortex (FIG. 2). Because NAA is present primarily in neurons, these metabolic changes reflect a loss or dysfunction of motor neurons. A decreased NAA/Cr and NAA/Cho ratio along the CST with the most pronounced reduction in the precentral gyrus and corona radiata has been shown in patients with ALS [58]. Similarly, a reduction in NAA from the pons and upper medulla has also been reported in ALS patients who have prominent UMN or bulbar signs [59].

The degree of NAA reduction varies significantly from one study to another, probably due to differences in spectral acquisition techniques, the severity of disease, definition of patient, control populations [15, 18, 56], among other factors. There is no consensus on which metabolite ratios accurately reflect UMN involvement $[18,19,56]$. While some studies have reported NAA/ Cho ratios as the most sensitive indicator of neuronal loss, others have concluded that $\mathrm{NAA} / \mathrm{Cr}$ ratio is the most optimal parameter in the diagnosis of ALS [18, 19, 56]. A moderate correlation between NAA concentration from the motor cortex and clinical manifestations (disease severity [15, 60], UMN signs [20], and maximum finger tapping rate [57]) has been reported in some studies. Bulbar-onset patients have lower NAA from the motor cortex compared with those of limb-onset patients 

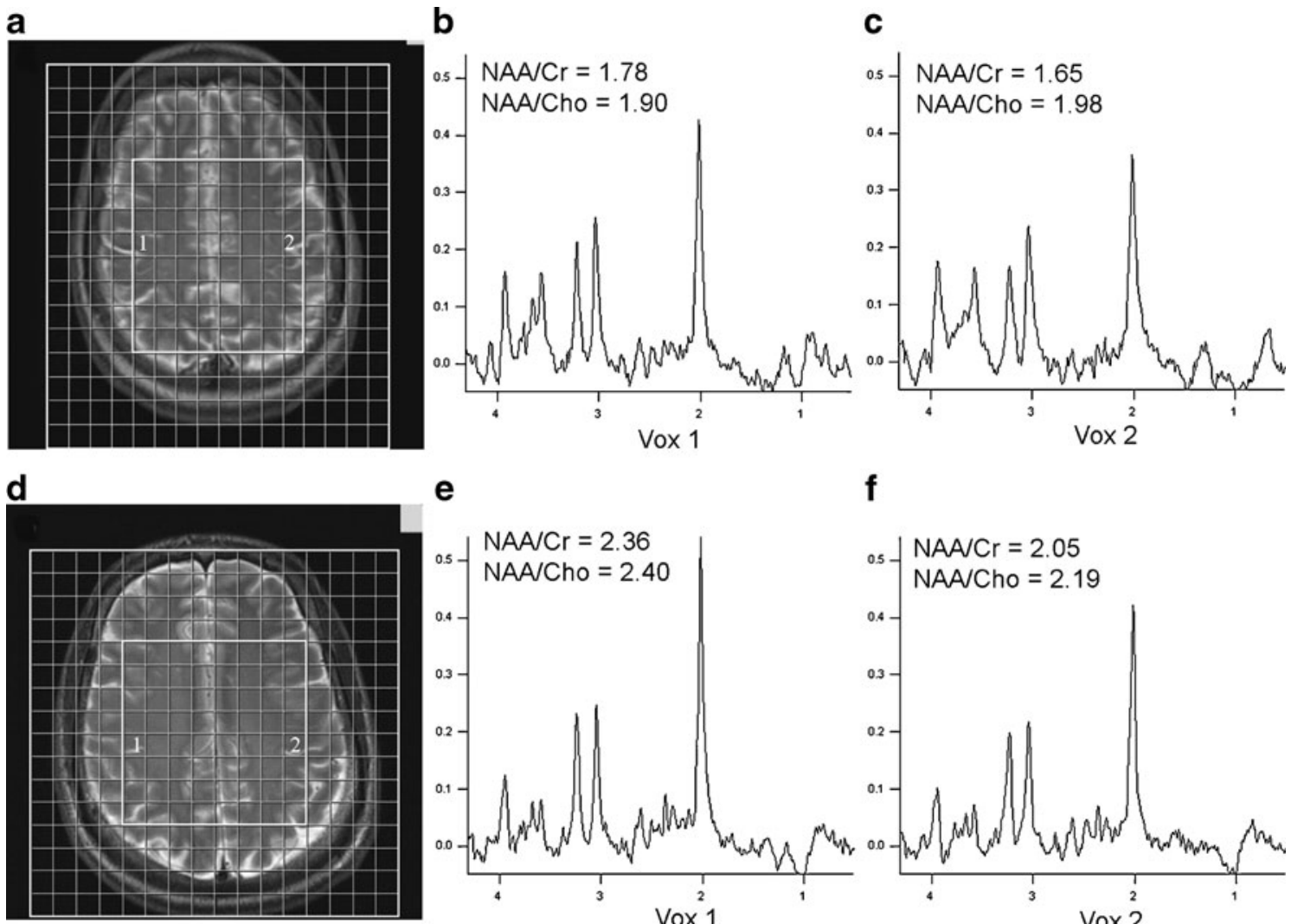

e

\section{f}
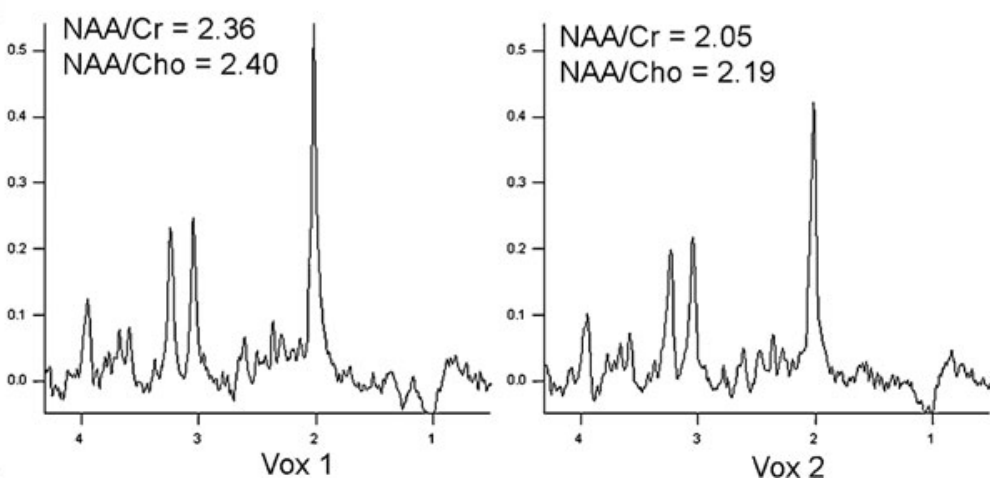

FIG. 2. Magnetic resonance (MR) images and spectra from 2-dimensional chemical shift imaging (CSI) spectroscopy of the motor cortex in a patient with amyotrophic lateral sclerosis (ALS) (a-c) and a control subject (d-f). Transverse T2-weighted (4000/85) MR images show the grid and volume of interest (rectangle) for the CSI study in the patient (a) and control subject (d). Representative spectra of the motor cortex from the (ALS) patient $(b, c)$ demonstrate reduced NAA/Cr and NAA/Cho ratios compared with the control subject $(e, f)$. NAA $=N$ acetyl aspartate, $\mathrm{Cho}=$ choline, $\mathrm{Cr}=$ creatine. Reproduced with permission from Wang et al. [15]. (High resolution version of this image is available in the electronic supplementary material.)

[61]. Furthermore, patients with a lower NAA/Cho ratio showed reduced survival [62].

In addition to decreased NAA, increased Cho has been reported in the PLIC in patients with ALS [56]. Increased $\mathrm{mI}$ has also been found in the motor cortex in ALS patients $[17,18]$. Using a higher field magnet (3 Tesla), Kalra et al. [63] reported that the NAA/mI ratio provided both better sensitivity and specificity for detecting ALS than individual metabolites and demonstrated the strongest correlation with disease severity.

In ALS, decreased NAA is not only restricted to the motor cortex and CST, but this reduction is also observed in premotor regions, the primary sensory cortex, and extramotor frontal regions with relative sparing of the parietal lobe [64]. A recent study demonstrated that alterations of NAA and Cho in ALS are correlated with each other [65]. This correlation is consistent with new pathological findings that diverse membrane proteins which give rise to increased Cho may contribute to motor neuron death [66].

MR spectroscopy can also be used to monitor treatment response. An increased $\mathrm{NAA} / \mathrm{Cr}$ ratio in the motor cortex has been observed in some studies after a short course of treatment with riluzole [67, 68]. However, no effect was seen after treatment with gabepentin [69]. Thus, it can be concluded that NAA has the potential to serve as a surrogate marker for therapeutic efficacy.

\section{PERFUSION IMAGING}

\section{Basic physics}

Perfusion is defined as the volume of blood that passes through a mass of tissue per unit time. Brain perfusion, also termed cerebral blood flow (CBF), is expressed in units of milliliters per gram per minute. All perfusion measurements require a diffusible or nondiffusible tracer whose transport in the blood can be measured. Perfusion imaging was pioneered with nuclear medicine techniques using positron emission tomography and single photon emission CT [70, 71].

The two most common methods for measuring tissue perfusion by MRI in the brain are dynamic susceptibility contrast-enhanced imaging, in which a bolus of a 
gadolinium-based contrast agent is injected and the response is observed in the form of a transient decrease in T2/T2*-weighted MRI signal, and arterial spin labeling (ASL), which utilizes arterial blood water as an endogenous diffusible tracer for measuring CBF. In ASL, CBF is measured by magnetically inverting the magnetization of water protons in the inflowing arteries and collecting the signal after the labeled blood water flows into the imaging region and diffuses into brain tissue. Because this method is entirely noninvasive and can be repeated rapidly, it has been widely used in assessing blood flow in patients with neurodegenerative diseases.

\section{Clinical application}

Previous studies using positron emission tomography or single photon emission CT demonstrated reduced regional cerebral metabolism or perfusion throughout the brain in patients with ALS [70-72]. The involved areas were not only limited to the sensormotor cortex, but also included extramotor regions, such as the premotor, prefrontal, cingulate, putamen, insular, and parietal cortex regions [70-73]. In contrast, patients with progressive muscular atrophy appeared to have normal or near-normal cerebral metabolism and $\mathrm{CBF}[74,75]$. A recent study in ALS showed similar findings using ASL perfusion [21]. These authors also found regional areas where increased ALS severity correlated with decreased gray matter perfusion, suggesting that measurement of brain perfusion may be another useful tool for monitoring disease progression and assessing treatment effects in ALS (FIG. 3).

\section{MAGNETIZATION TRANSFER IMAGING}

\section{Basic physics}

Magnetization transfer imaging (MTI) is based on the exchange of magnetization between protons in free water and protons that are bound to macromolecules. The magnitude of the effect depends on the relative water and macromolecular concentration, surface chemistry and biophysical dynamics of macromolecules and may be quantified by measuring the MT ratio (MTR). MTR can be calculated according to the following equation:

$$
\mathrm{MTR}=\left[\left(\mathrm{M}_{0}-\mathrm{Ms}\right) / \mathrm{M}_{0}\right] \times 100
$$

where $\mathrm{M}_{0}$ is the measured signal intensity from images without the magnetization transfer pulse, and Ms is the measured signal intensity with the magnetization transfer pulse [76]. In the brain, macromolecules contributing to the MT effect include the cholesterol component of myelin, cerebrosides, and phospholipids. MT imaging can be used to determine the local tissue status by assessing MTR values from representative ROIs or by performing MTR histogram analysis of the whole brain.
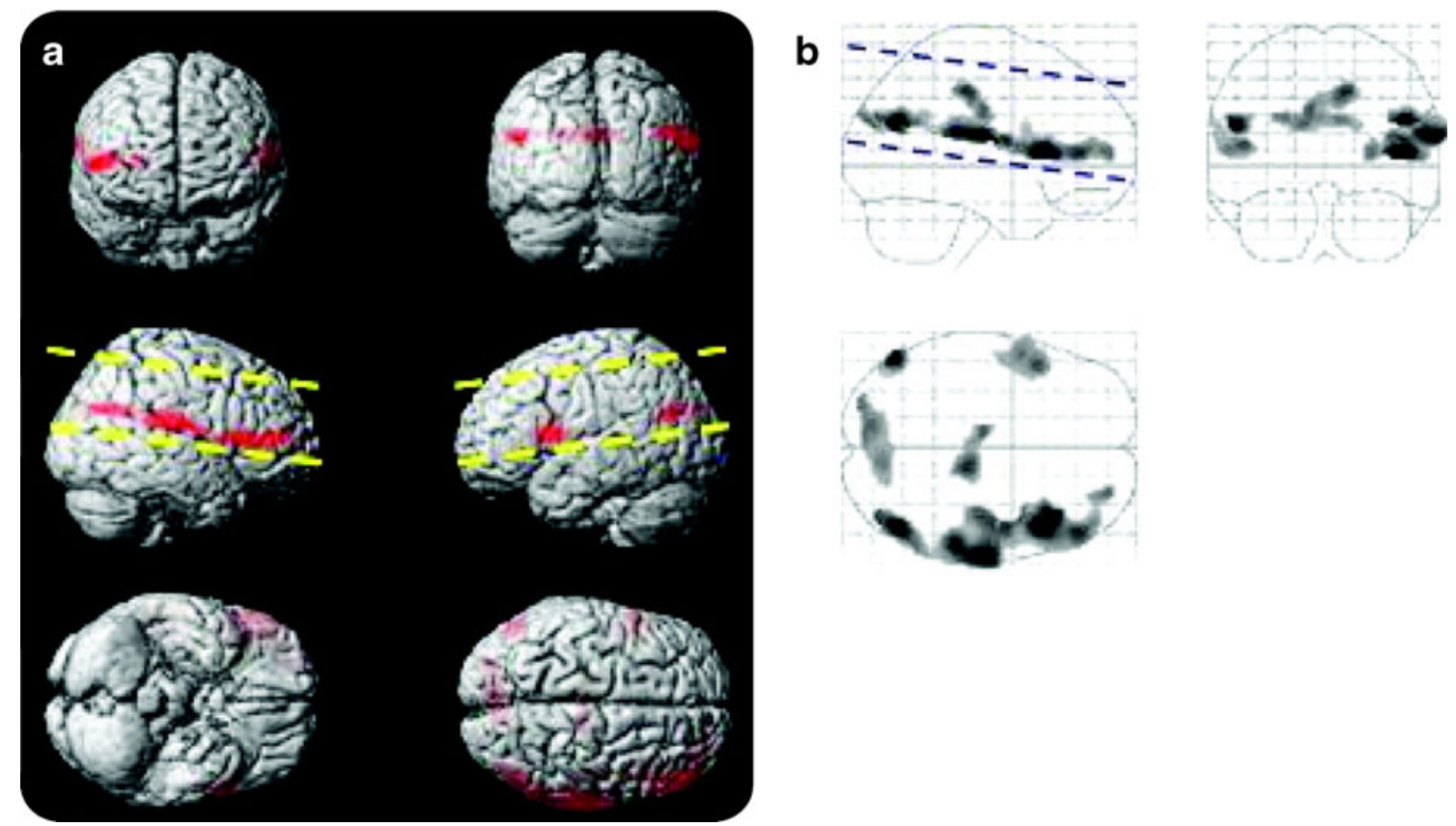

FIG. 3. Correlation between the ALS functional rating scale (ALSFRS) and ASL. The figure shows voxel clusters in red where ALSFRS has a positive correlation with ASL perfusion. The images were individually oriented so that the left brain was ipsilateral to the side of the more significant weakness seen clinically. Voxels were only included if they were in clusters of at least 100 voxels $(p<0.001)$. The images in (a) project these voxels onto a 3-dimensional rendering of the Montreal Neurological Institute atlas. The images in (b) display these same data as a maximum intensity projection on a transparent "glass brain." The dashed lines indicate the extent of the brain that was covered by the ASL perfusion images. Reproduced with permission from Rule et al. [21]. (High resolution version of this image is available in the electronic supplementary material.) 
Typically, a low MTR indicates damage to myelin or to the axonal membrane.

\section{Clinical application}

MTI has been shown to be able to detect the pathology of the CST in ALS. Da Rocha et al. [22] reported hyperintensity on T1-weighted spin-echo magnetization transfer contrast images along the CST in $80 \%$ of patients with ALS. Some studies have also demonstrated a reduction in MTR from the internal capsule in patients with ALS [77, 78], implying the presence of tissue damage in these areas. The results of these studies suggest that MTI facilitates the detection of CST degeneration.

\section{FUNCTIONAL MRI}

Functional MRI provides high-resolution, noninvasive estimates of neural activity. When a neural event occurs in a region of the brain, despite the increase in oxygen consumption, there is a subsequent increase in local blood flow that paradoxically results in a decrease in the concentration of deoxygenated hemoglobin in the local microvasculature of the activated region. Since deoxyhemoglobin is paramagnetic, it alters the $\mathrm{T} 2 *$-weighted magnetic resonance image signal. Therefore, this change leads to an increase in the fMRI signal that has been called the blood oxygenated level-dependent effect [23, 79].

A general pattern of cortical reorganization was found for motor function in patients with ALS when compared with that seen in normal subjects [24], with increased activation of the contralateral sensorimotor cortex, supplementary motor area, basal ganglia, and cerebellum demonstrated on fMR images during motor tasks $[24,25$, 80, 81]. Increased sensorimotor activation has also been reported in the brain ipsilateral to the movement [80]. In addition, reduced activation in the dorsolateral prefrontal cortex has been observed, providing further evidence for the existence of frontal lobe function deficits in patients with ALS [81].

\section{CONCLUSIONS}

Advanced MRI techniques, such as DTI, MRS, perfusion, MTI and fMRI, are increasingly being used to investigate the anatomic and functional changes in ALS. These novel imaging modalities provide insight into the pathophysiological process in ALS and may play a role in accurate identification of UMN pathology in ALS. Further investigation is needed to determine and to compare the relative utility of these various neuroimaging techniques. New acquisition schemes and analysis procedures require standardization and optimization.
Acknowledgments: This study was supported by NIH grant 1R21NS063111 (J.H.W.)

\section{REFERENCES}

1. Rowland LP. Diagnosis of amyotrophic lateral sclerosis. J Neurol Sci 1998;160 (Suppl 1):S6-24.

2. Ravits JM, La Spada AR. ALS motor phenotype heterogeneity, focality, and spread: deconstructing motor neuron degeneration. Neurology 2009;73:805-811.

3. Turner MR, Kiernan MC, Leigh PN, Talbot K. Biomarkers in amyotrophic lateral sclerosis. Lancet Neurol 2009;8:94-109.

4. Rowland LP, Shneider NA. Amyotrophic lateral sclerosis. N Engl J Med 2001;344:1688-1700.

5. Abrahams S, Goldstein LH, Suckling J, et al. Frontotemporal white matter changes in amyotrophic lateral sclerosis. J Neurol 2005;252:321-331.

6. Nakano I. Frontotemporal dementia with motor neuron disease (amyotrophic lateral sclerosis with dementia). Neuropathology 2000;20:68-75.

7. Chan S, Kaufmann P, Shungu DC, Mitsumoto H. Amyotrophic lateral sclerosis and primary lateral sclerosis: evidence-based diagnostic evaluation of the upper motor neuron. Neuroimaging Clin N Am 2003;13:307-326.

8. Hong YH, Lee KW, Sung JJ, Chang KH, Song IC. Diffusion tensor MRI as a diagnostic tool of upper motor neuron involvement in amyotrophic lateral sclerosis. J Neurol Sci 2004;227:73-78.

9. Ellis CM, Simmons A, Jones DK, et al. Diffusion tensor MRI assesses corticospinal tract damage in ALS. Neurology 1999;53:1051-1058.

10. Graham JM, Papadakis N, Evans J, et al. Diffusion tensor imaging for the assessment of upper motor neuron integrity in ALS. Neurology 2004;63:2111-2119.

11. Sach M, Winkler G, Glauche V, et al. Diffusion tensor MRI of early upper motor neuron involvement in amyotrophic lateral sclerosis. Brain 2004; 127:340-350.

12. Toosy AT, Werring DJ, Orrell RW, et al. Diffusion tensor imaging detects corticospinal tract involvement at multiple levels in amyotrophic lateral sclerosis. J Neurol Neurosurg Psychiatry 2003; $74: 1250-1257$

13. Ulug AM, Grunewald T, Lin MT, et al. Diffusion tensor imaging in the diagnosis of primary lateral sclerosis. J Magn Reson Imaging 2004;19:34-39.

14. Yin H, Lim CC, Ma L, et al. Combined MR spectroscopic imaging and diffusion tensor MRI visualizes corticospinal tract degeneration in amyotrophic lateral sclerosis. J Neurol 2004;251:1249-1254.

15. Wang S, Poptani H, Woo JH, et al. Amyotrophic lateral sclerosis: diffusion-tensor and chemical shift MR imaging at 3.0T. Radiology 2006;239:831-838.

16. Wang S, Poptani H, Bilello M, et al. Diffusion tensor imaging in amyotrophic lateral sclerosis: volumetric analysis of the corticospinal tract. AJNR Am J Neuroradiol 2006;27:1234-1238.

17. Block W, Karitzky J, Traber F, et al. Proton magnetic resonance spectroscopy of the primary motor cortex in patients with motor neuron disease: subgroup analysis and follow-up measurements. Arch Neurol 1998;55:931-936.

18. Bowen BC, Pattany PM, Bradley WG, et al. MR imaging and localized proton spectroscopy of the precentral gyrus in amyotrophic lateral sclerosis. AJNR Am J Neuroradiol 2000;21:647-658.

19. Chan S, Shungu DC, Douglas-Akinwande A, Lange DJ, Rowland LP. Motor neuron diseases: comparison of single-voxel proton MR spectroscopy of the motor cortex with MR imaging of the brain. Radiology 1999;212:763-769.

20. Kaufmann P, Pullman SL, Shungu DC, et al. Objective tests for upper motor neuron involvement in amyotrophic lateral sclerosis (ALS). Neurology 2004;62:1753-1757.

21. Rule RR, Schuff N, Miller RG, Weiner MW. Gray matter perfusion correlates with disease severity in ALS. Neurology 2010;74:821827.

22. da Rocha AJ, Oliveira AS, Fonseca RB, et al. Detection of corticospinal tract compromise in amyotrophic lateral sclerosis with 
brain MR imaging: relevance of the T1-weighted spin-echo magnetization transfer contrast sequence. AJNR Am J Neuroradiol 2004;25:1509-1515.

23. Brooks BR, Bushara K, Khan A, et al. Functional magnetic resonance imaging (fMRI) clinical studies in ALS-paradigms, problems and promises. Amyotroph Lateral Scler Other Motor Neuron Disord 2000;1 (Suppl 2):S23-32.

24. Konrad C, Henningsen H, Bremer J, et al. Pattern of cortical reorganization in amyotrophic lateral sclerosis: a functional magnetic resonance imaging study. Exp Brain Res 2002;143:51-56.

25. Konrad C, Jansen A, Henningsen H, et al. Subcortical reorganization in amyotrophic lateral sclerosis. Exp Brain Res 2006;172:361-369.

26. Agosta F, Chio A, Cosottini M, et al. The present and the future of neuroimaging in amyotrophic lateral sclerosis. AJNR Am J Neuroradiol. 2010;31:1769-1777

27. Wang S, Melhem ER. Amyotrophic lateral sclerosis and primary lateral sclerosis: The role of diffusion tensor imaging and other advanced MR-based techniques as objective upper motor neuron markers. Ann N Y Acad Sci 2005;1064:6131:1769-1777-77.

28. Hecht MJ, Fellner F, Fellner C, et al. MRI-FLAIR images of the head show corticospinal tract alterations in ALS patients more frequently than T2-, T1- and proton-density-weighted images. J Neurol Sci 2001;186:37-44.

29. Zhang L, Ulug AM, Zimmerman RD, et al. The diagnostic utility of FLAIR imaging in clinically verified amyotrophic lateral sclerosis. J Magn Reson Imaging 2003;17:521-527.

30. Karantanas AH. Amyotrophic lateral sclerosis: unilateral T2-shortening on MRI. Comput Med Imaging Graph 1998;22:353-355.

31. Oba H, Araki T, Ohtomo K, et al. Amyotrophic lateral sclerosis: T2 shortening in motor cortex at MR imaging. Radiology 1993;189:843-846.

32. Grosskreutz J, Peschel T, Unrath A, et al. Whole brain-based computerized neuroimaging in ALS and other motor neuron disorders. Amyotroph Lateral Scler 2008;9:238-248.

33. Ashburner J, Friston KJ. Voxel-based morphometry-the methods. Neuroimage 2000;11:805-821.

34. Kassubek J, Unrath A, Huppertz HJ, et al. Global brain atrophy and corticospinal tract alterations in ALS, as investigated by voxelbased morphometry of 3-D MRI. Amyotroph Lateral Scler Other Motor Neuron Disord 2005;6:213-220.

35. Mezzapesa DM, Ceccarelli A, Dicuonzo F, et al. Whole-brain and regional brain atrophy in amyotrophic lateral sclerosis. AJNR Am J Neuroradiol 2007;28:255-259.

36. Agosta F, Pagani E, Rocca MA, et al. Voxel-based morphometry study of brain volumetry and diffusivity in amyotrophic lateral sclerosis patients with mild disability. Hum Brain Mapp 2007;28:1430-1438.

37. Chang JL, Lomen-Hoerth C, Murphy J, et al. A voxel-based morphometry study of patterns of brain atrophy in ALS and ALS/ FTLD. Neurology 2005;65:75-80.

38. Beaulieu $C$. The basis of anisotropic water diffusion in the nervous system - a technical review. NMR Biomed 2002;15:435-455.

39. Basser PJ, Pierpaoli C. Microstructural and physiological features of tissues elucidated by quantitative-diffusion-tensor MRI. J Magn Reson B 1996;111:209-219.

40. Ciccarelli O, Behrens TE, Altmann DR, et al. Probabilistic diffusion tractography: a potential tool to assess the rate of disease progression in amyotrophic lateral sclerosis. Brain 2006;129:1859-1871.

41. Mori S, Crain BJ, Chacko VP, van Zijl PC. Three-dimensional tracking of axonal projections in the brain by magnetic resonance imaging. Ann Neurol 1999;45:265-269.

42. Stieltjes B, Kaufmann WE, van Zijl PC, et al. Diffusion tensor imaging and axonal tracking in the human brainstem. Neuroimage 2001;14:723-735.

43. Behrens TE, Johansen-Berg H, Woolrich MW, et al. Non-invasive mapping of connections between human thalamus and cortex using diffusion imaging. Nat Neurosci 2003;6:750-757.

44. Hong YH, Sung JJ, Kim SM, et al. Diffusion tensor tractographybased analysis of the pyramidal tract in patients with amyotrophic lateral sclerosis. J Neuroimaging 2008;18:282-287.

45. Sage CA, Peeters RR, Gorner A, Robberecht W, Sunaert S. Quantitative diffusion tensor imaging in amyotrophic lateral sclerosis. Neuroimage 2007;34:486-499.
46. Abe O, Yamada H, Masutani Y, et al. Amyotrophic lateral sclerosis: diffusion tensor tractography and voxel-based analysis. NMR Biomed 2004; 17:411-416.

47. Smith SM, Jenkinson M, Johansen-Berg H, et al. Tract-based spatial statistics: voxelwise analysis of multi-subject diffusion data. Neuroimage 2006;31:1487-1505.

48. Ciccarelli O, Behrens TE, Johansen-Berg H, et al. Investigation of white matter pathology in ALS and PLS using tract-based spatial statistics. Hum Brain Mapp 2009;30:615-624.

49. Sage CA, Van Hecke W, Peeters R, et al. Quantitative diffusion tensor imaging in amyotrophic lateral sclerosis: revisited. Hum Brain Mapp 2009;30:3657-3675.

50. Agosta F, Pagani E, Petrolini M, et al. Assessment of white matter tract damage in patients with Amyotrophic Lateral Sclerosis: A diffusion tensor MR imaging tractography study. AJNR Am J Neuroradiol 2010;31:1457-1461

51. Castillo M, Kwock L, Mukherji SK. Clinical applications of proton MR spectroscopy. AJNR Am J Neuroradiol 1996;17:1-15.

52. Maudsley AA, Darkazanli A, Alger JR, et al. Comprehensive processing, display and analysis for in vivo MR spectroscopic imaging. NMR Biomed 2006;19:492-503.

53. Maudsley AA, Domenig C, Govind V, et al. Mapping of brain metabolite distributions by volumetric proton MR spectroscopic imaging (MRSI). Magn Reson Med 2009;61:548-559.

54. Bradley WG, Bowen BC, Pattany PM, Rotta F. 1H-magnetic resonance spectroscopy in amyotrophic lateral sclerosis. J Neurol Sci 1999;169:84-86.

55. Pohl C, Block W, Karitzky J, et al. Proton magnetic resonance spectroscopy of the motor cortex in 70 patients with amyotrophic lateral sclerosis. Arch Neurol 2001;58:729-735.

56. Schuff N, Rooney WD, Miller R, et al. Reanalysis of multislice (1) $\mathrm{H}$ MRSI in amyotrophic lateral sclerosis. Magn Reson Med 2001;45:513-516.

57. Rooney WD, Miller RG, Gelinas D, et al. Decreased N-acetylaspartate in motor cortex and corticospinal tract in ALS. Neurology 1998;50:1800-1805.

58. Pyra T, Hui B, Hanstock C, et al. Combined structural and neurochemical evaluation of the corticospinal tract in amyotrophic lateral sclerosis. Amyotroph Lateral Scler;11:157-165.

59. Cwik VA, Hanstock CC, Allen PS, Martin WR. Estimation of brainstem neuronal loss in amyotrophic lateral sclerosis with in vivo proton magnetic resonance spectroscopy. Neurology 1998;50:7-277.

60. Mitsumoto H, Ulug AM, Pullman SL, et al. Quantitative objective markers for upper and lower motor neuron dysfunction in ALS. Neurology 2007;68:1402-1410.

61. Ellis CM, Simmons A, Andrews C, et al. A proton magnetic resonance spectroscopic study in ALS: correlation with clinical findings. Neurology 1998;51:1104-1109.

62. Kalra S, Vitale A, Cashman NR, Genge A, Arnold DL. Cerebral degeneration predicts survival in amyotrophic lateral sclerosis. J Neurol Neurosurg Psychiatry 2006;77:1253-1255.

63. Kalra S, Hanstock CC, Martin WR, Allen PS, Johnston WS Detection of cerebral degeneration in amyotrophic lateral sclerosis using high-field magnetic resonance spectroscopy. Arch Neurol 2006;63:1144-148.

64. Pioro EP, Antel JP, Cashman NR, Arnold DL. Detection of cortical neuron loss in motor neuron disease by proton magnetic resonance spectroscopic imaging in vivo. Neurology 1994;44:1933-1938.

65. Young K, Govind V, Sharma K, et al. Multivariate statistical mapping of spectroscopic imaging data. Magn Reson Med;63:20-24.

66. Masters CL, Cappai R, Barnham KJ, Villemagne VL. Molecular mechanisms for Alzheimer's disease: implications for neuroimaging and therapeutics. J Neurochem 2006;97:1700-1725.

67. Kalra S, Cashman NR, Genge A, Arnold DL. Recovery of Nacetylaspartate in corticomotor neurons of patients with ALS after riluzole therapy. Neuroreport 1998;9:1757-1761.

68. Kalra S, Tai P, Genge A, Arnold DL. Rapid improvement in cortical neuronal integrity in amyotrophic lateral sclerosis detected by proton magnetic resonance spectroscopic imaging. J Neurol 2006;253:1060-1063

69. Kalra S, Cashman NR, Caramanos Z, Genge A, Arnold DL. Gabapentin therapy for amyotrophic lateral sclerosis: lack of 
improvement in neuronal integrity shown by MR spectroscopy. AJNR Am J Neuroradiol 2003;24:476-480.

70. Hatazawa J, Brooks RA, Dalakas MC, Mansi L, Di Chiro G. Cortical motor-sensory hypometabolism in amyotrophic lateral sclerosis: a PET study. J Comput Assist Tomogr 1988;12:630-636.

71. Udaka F, Sawada H, Seriu N, et al. MRI and SPECT findings in amyotrophic lateral sclerosis. Demonstration of upper motor neurone involvement by clinical neuroimaging. Neuroradiology 1992;34:389-393.

72. Habert MO, Lacomblez L, Maksud P, et al. Brain perfusion imaging in amyotrophic lateral sclerosis: extent of cortical changes according to the severity and topography of motor impairment. Amyotroph Lateral Scler 2007;8:9-15.

73. Kew JJ, Leigh PN, Playford ED, et al. Cortical function in amyotrophic lateral sclerosis. A positron emission tomography study. Brain 1993;116:655-680.

74. Dalakas MC, Hatazawa J, Brooks RA, Di Chiro G. Lowered cerebral glucose utilization in amyotrophic lateral sclerosis. Ann Neurol 1987;22:580-586.

75. Kew JJ, Brooks DJ, Passingham RE, et al. Cortical function in progressive lower motor neuron disorders and amyotrophic lateral sclerosis: a comparative PET study. Neurology 1994;44:11011110 .

76. Wolff SD, Balaban RS. Magnetization transfer imaging: practical aspects and clinical applications. Radiology 1994;192:593-599.

77. Kato Y, Matsumura K, Kinosada Y, et al. Detection of pyramidal tract lesions in amyotrophic lateral sclerosis with magnetizationtransfer measurements. AJNR Am J Neuroradiol 1997;18:15411547.

78. Tanabe JL, Vermathen M, Miller R, et al. Reduced MTR in the corticospinal tract and normal T2 in amyotrophic lateral sclerosis. Magn Reson Imaging 1998;16:1163-1169.

79. Ogawa S, Menon RS, Tank DW, et al. Functional brain mapping by blood oxygenation level-dependent contrast magnetic resonance imaging. A comparison of signal characteristics with a biophysical model. Biophys J 1993;64:803-812.

80. Schoenfeld MA, Tempelmann C, Gaul C, et al. Functional motor compensation in amyotrophic lateral sclerosis. J Neurol 2005;252:944-952.

81. Stanton BR, Williams VC, Leigh PN, et al. Altered cortical activation during a motor task in ALS. Evidence for involvement of central pathways. J Neurol 2007;254:1260-1267. 